\title{
Simulation study of local thermal runaway of 18650 lithium battery module under multi-point thermal trigger
}

\author{
Yu Zhang ${ }^{1,2, a}$, Guixiong Liu ${ }^{1, b^{*}}$, Daqiang Deng ${ }^{2, c}$ \\ ${ }^{1}$ School of Mechanical and Automotive Engineering, South China University of Technology, Tianhe, Guangzhou, Guangdong, China \\ ${ }^{2}$ School of Mechanical Engineering, Guangzhou College of South China University of Technology, Huadu, Guangzhou, Guangdong, \\ China
}

\begin{abstract}
The thermal runaway of lithium power battery is the key problem of battery safety, according to the standard SAE J2464-2009 single point heating key position, the proposed multi-point trigger based thermal runaway of lithium power battery module simulation method and battery module thermal runaway battery monomer ratio $P_{N}$, thermal runaway trigger time $t_{n}$ two indicators, the thermal runaway rule of 18650 lithium power battery module under different trigger position, trigger points. The results show that considering the external thermal insulation conditions of the power lithium battery module, the geometric angular position in the module is the most dangerous position, and the number of thermal trigger points is positively correlated with $P_{N}$.
\end{abstract}

\section{Introduction}

Thermal runaway refers to the uncontrolled rise of battery temperature caused by a chain reaction of heat release from the battery monomer, and the safety of lithium-ion battery as the core component of electric vehicles, which restricts the development of electric vehicles. Power battery module as an important component of the battery system in electric vehicles, when the collision and other accidents, the presence of battery modules in multiple locations in the battery is squeezed, resulting in multiple points of overheating, which in turn triggers the battery module and even the entire battery system to produce thermal runaway situation.

At present, scholars at home and abroad have proposed a variety of methods for the study of thermal runaway behavior of power batteries, Tsinghua University Feng Xuning (2018) predicted the temperature and pressure drop in the thermal runaway of cylindrical battery monomer by establishing a two-dimensional electrochemical-thermal coupling model, which has a high predictive power but cannot represent the thermal runaway process of the battery visually through a threedimensional diagram[1]; Sara (2018), Sorbonne University, France Using Multiphysics coupled model to study the thermal runaway mechanism of lithium battery monomer, calculations showed that thickening the SEI film could delay the occurrence of thermal runaway of the battery[2]. Ji Changwei et al. (2019) of Beijing University of Technology conducted a central position heating test on a battery module composed of 32650 battery monomers to study the rule of triggering thermal runaway of the battery module as a whole under single-point thermal triggering conditions, but did not involve the overheating of the module at multiple points to generate thermal runaway [3]. The above papers have analyzed and studied the thermal runaway behavior of the power battery monomer and the module under single-point thermal triggering, but they do not involve the evaluation of the dangerous location under single-point thermal triggering and the thermal runaway caused by multi-point thermal triggering in the battery module. Different from the above literature, this paper takes the square module composed of cylindrical lithium battery 18650 monomer as the object of study, constructs the $3 \mathrm{D}$ thermal abuse model, performs the single point thermal trigger for the power battery module at the five recommended locations in the American standard SAE J2464-2009, and then calculates the percentage and time of thermal runaway of the battery monomer in the module to obtain the most likely location of thermal runaway in the module. Secondly, on the basis of multi-point thermal trigger, we study the thermal runaway rule of the module under different locations and different number of trigger points.

\section{Heat generation and heat transfer mechanism of thermal runaway battery}

Thermal runaway of the power battery can occur under abusive conditions, which mainly include mechanical abuse, electrical abuse and thermal abuse, and both mechanical abuse and electrical abuse will eventually lead to high temperature of the battery, thus generating thermal abuse and causing thermal runaway of the battery [4,5], therefore, the impact of thermal abuse on the battery is more serious.

The heat generated by the lithium-ion battery includes

aemail: 201821003079@mail.scut.edu.cn,

*Guixiong Liu: bemail: megxliu@scut.edu.Cn, cemail: dengdq@139.com. 
heat of reaction, heat of polarization internal resistance, ohmic internal resistance heat, and heat of secondary reaction. The heat of reaction, internal resistance heat of polarization and ohmic internal resistance heat is due to the electrochemical reaction of lithium ion batteries in the charge and discharge state to generate heat, but this paper studies the thermal behavior of the battery under the conditions of thermal abuse, so it is not considered. When the battery temperature is too high, the active materials inside the battery decompose, and a series of side reactions generate a large amount of heat, inducing the battery thermal runaway. The types of battery thermal runaway side reactions include SEI membrane decomposition, negative electrode and electrolyte reaction, positive electrode and electrolyte reaction, and electrolyte decomposition reaction. Set $Q_{p e}, Q_{n e}, Q_{\text {sei }}$, and $Q_{\text {ele }}$ as the heat production rates $\left(\mathrm{W} / \mathrm{m}^{3}\right)$ for the reactions of positive electrode and electrolyte, negative electrode and electrolyte, SEI film decomposition, and electrolyte decomposition, respectively, then the total heat production rate $\mathrm{Q}$ of the battery in thermal abuse thermal runaway is [6-9].

$$
Q=Q_{p e}+Q_{n e}+Q_{\text {sei }}+Q_{\text {ele }}
$$

(1) Reaction between anode and electrolyte. If $H_{p e}$ is the heat released per unit material $(\mathrm{J} / \mathrm{kg}), W_{p}$ is the content of active material $(\mathrm{kg} / \mathrm{m} 3), A_{p e}$ is the frequency factor (s$1), E_{a, p e}$ is the activation energy of reaction $(\mathrm{J} / \mathrm{mol}), m_{p e l}$, $m_{p e 2}$ is the number of reaction stages, and $b$ is the ratio of the reacted anode material to all anode materials, then the heat production rate of the reaction between anode and electrolyte is as follows.

$$
Q_{p e}=H_{p e} \cdot W_{p} \cdot A_{p e} \cdot b^{m_{p e 1}}(1-b)^{m_{p e 2}} \exp \left(-\frac{E_{a, p e}}{R T}\right)
$$

(2) Reaction between cathode and electrolyte. If $H_{n e}$ is the amount of heat released per unit material $(\mathrm{J} / \mathrm{kg}), W_{c}$ is the carbon content $(\mathrm{kg} / \mathrm{m} 3), A_{n e}$ is the frequency factor $\left(\mathrm{s}^{-}\right.$ $\left.{ }^{1}\right), E_{a, n e}$ is the activation energy of reaction $(\mathrm{J} / \mathrm{mol}), C_{n e}$ is the ratio of lithium embedded in carbon cathode to electrolyte, $m_{n e}$ is the number of reaction stages, $t_{s e i}$ is the ratio of sei membrane to active material, then the heat production rate of the reaction between cathode and electrolyte is as follows.

$$
Q_{n e}=H_{n e} \cdot W_{c} \cdot A_{n e} \exp \left(-\frac{E_{a, n e}}{R T}\right) \mathrm{C}_{n e}^{m_{n e}} \exp \left(-\frac{t_{\text {sei }}}{t_{\text {sei }, r e f}}\right)
$$

(3) SEI membrane decomposition. Set $H_{\text {sei }}$ as the unit material heat release $(\mathrm{J} / \mathrm{kg}), W_{c}$ is the carbon content $(\mathrm{kg} / \mathrm{m} 3), \mathrm{R}=8.314 \mathrm{~J} /(\mathrm{mol}-\mathrm{K})$ as the gas reaction constant, $A_{\text {sei }}$ is the frequency factor (s-1); $E_{a, s e i}$ is the activation energy of the reaction, $(\mathrm{J} / \mathrm{mol}) ; m_{s e i}$ refers to the number of reaction stages, $C_{s e i}$ is the proportion of unstable lithium, then the heat production rate of SEI membrane decomposition is.

$$
Q_{s e i}=H_{s e i} \cdot W_{c} \cdot A_{s e i} \exp \left(-\frac{E_{a, s e i}}{R T}\right) C_{s e i}^{m_{s e i}}
$$

(4) Electrolyte decomposition reaction. If $H_{e}$ is the heat released per unit material reaction $(\mathrm{J} / \mathrm{kg}), W_{e}$ is the electrolyte content $(\mathrm{kg} / \mathrm{m} 3), A_{e}$ is the frequency factor $\left(\mathrm{s}^{-}\right.$ $\left.{ }^{1}\right), E_{a, e}$ is the reaction activation energy $(\mathrm{J} / \mathrm{mol}), m_{e}$ is the number of reaction stages, $C_{e}$ is the ratio of the remaining electrolyte to the total electrolyte material, then the heat production rate of the electrolyte decomposition reaction is as follows.

$$
Q_{\text {ele }}=H_{e} \cdot W_{e} \cdot A_{e} \exp \left(-\frac{E_{a, e}}{R T}\right) C_{e}^{m_{e}}
$$

In order to simplify the model and reduce the amount of calculation, the simulation considers heat conduction and air convection as the means of heat exchange in the model (heat radiation is less and can be ignored in practical application). Set $\lambda$ is the thermal conductivity, $\delta$ is the mutual distance between cells, $A$ is the contact area between the three-dimensional model and air, $h$ is the convective heat transfer coefficient of the battery and air, $\Delta T$ is the temperature difference between the battery and air fluid, then heat conduction $\mathrm{q} 1$, thermal convection q2 are.

$$
q_{1}=\lambda \frac{\Delta T}{\delta}, q_{2}=A h \cdot \Delta T
$$

\section{Battery thermal abuse simulation model and evaluation index}

\subsection{Simulation modeling}

The module uses 18650 lithium ternary lithium power battery monomer in a $3 \times 4$ arrangement constituted by the monomer spacing of $0.3 \mathrm{~mm}$, Figure 1 shows the geometric model of the power battery module and heating position diagram. The red dot marks the location of the U.S. standard SAE J2464-2009 recommended thermal runaway trigger position, including the battery module geometric angle position (point-1), the edge of the midpoint position (point-2), the face center position (point-3), the B-side from the opposite $1 / 4$ position (point4), C-side from the opposite $1 / 4$ position (point-5). The thermal abuse model $[10,11]$ is based on the heat generation mechanism of the four side reactions in equations (2)-(5), and the relationship between the heat emitted from the side reactions and the temperature is expressed by differential equations, calculated and added to the 12 battery monomers, and the temperature change curve of the lithium battery model under thermal abuse is calculated and obtained by COMSOL.

The quality and size of the grid of each part of the model has a great impact on the amount of later calculations and the accuracy of the results [12]. The smaller the grid division, the more units in the grid, the higher the overall quality of the grid, but the calculation volume will increase, requiring more memory for calculation; on the other hand, the larger the grid, the fewer the number of units included in the grid, the poorer the quality of the grid, the calculation may not converge. Figure 2 shows the grid division result of the lithium power battery module, using the Sagittal and free tetrahedral grids for the battery and shell parts, respectively, to obtain a better-quality grid. 


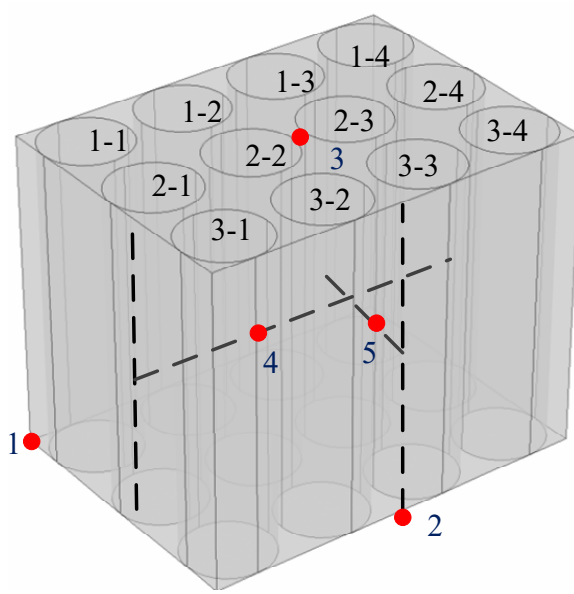

Figure 1. Power cell module geometry model and heating position diagram

\subsection{Thermal runaway evaluation index}

The national standard "Safety Requirements for Lithiumion Power Battery for Electric Vehicles" points out that when the lithium battery temperature reaches the manufacturer's maximum temperature of $60{ }^{\circ} \mathrm{C}$, the temperature rise rate is greater than $1{ }^{\circ} \mathrm{C} / \mathrm{s}$ and last for more than $3 \mathrm{~s}$, the battery can be judged thermal runaway, in order to better describe the thermal runaway law of lithium-ion battery monomer and module, the definition of indicators of thermal runaway battery percentage $P_{N}$, thermal runaway time $t_{n}$ two indicators to evaluate the lithium battery.

(1) Percentage of thermal runaway batteries $P_{N}$. It is the ratio of the number of thermal runaway monomers $N$ and the total number of monomers $N_{I}$ after the end of thermal runaway triggered in the power cell module battery. Characterizes the degree of thermal runaway of the battery module, the larger the $P_{N}$., the greater the degree of thermal runaway, the higher the degree of danger. That is:

$$
P_{N}=\frac{N}{N_{1}} \times 100 \%
$$

(2) Thermal runaway time $t_{n}$. It is the time from the start of thermal trigger to the occurrence of thermal runaway of the first monomer in the power cell module, where $n$ is the number of trigger points. The speed of thermal runaway of the battery module, the smaller $t_{n}$ is, the faster and more dangerous it is.

\section{Simulation experiments and results analysis}

\subsection{Simulation of thermal runaway of module under single point trigger}

In order to study the thermal runaway law at module level with different trigger points, the same point-like trigger heat source is applied at the five trigger points

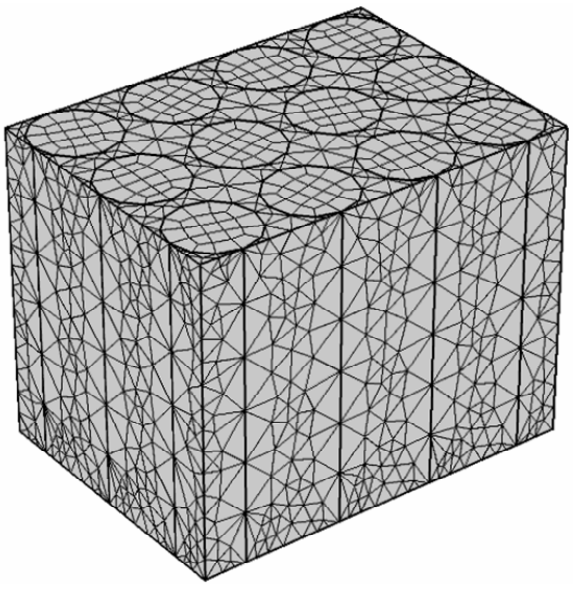

Fig. 2. Grid division result of Li-ion battery module

recommended by SAE J2464-2009, after simulation, it is judged whether each monomer in the model reaches the critical condition of thermal runaway, and then the $P_{N}$ of thermal runaway is calculated, and the $P_{N}$ of thermal runaway is compared with the $P_{N}$ of thermal runaway and the thermal runaway time $t_{n}$ at different trigger points.

It can be seen that when the thermal trigger position is point-2,3,4,5, all of them can trigger the thermal runaway of the battery module. Among them, at point-1 (geometric angle position), the thermal runaway time $t_{n}$ is the shortest, the percentage of thermal runaway cells is the largest, i.e. at point-1, the thermal runaway time is the fastest, the fastest diffusion and the most thermal runaway monomer; at point-2 (edge midpoint position), the thermal runaway time $t_{n}$ is the second, the percentage of thermal runaway cells is the same as that at point-1, the most thermal runaway monomer. When triggered at point-3,4,5, the thermal runaway time $t_{n}$ is longer and not much different, but the percentage of the thermal runaway cell at point- 4 is the same as that at point- 1,2 .

Table 1 shows the results of single-point thermal trigger $P_{N}$ and $t_{n}$ at different positions at the module level. It can be seen that when the thermal trigger position is at points $-2,3,4$, and 5 , the battery module can cause thermal runaway. Among them, when thermally triggered at point -1 (geometric angle position), the thermal runaway time $t_{n}$ is the shortest, and the thermal runaway battery accounts for the largest $P_{N}$. That is, the thermal trigger is performed at point -1 , and the battery module generates the fastest thermal runaway time. The diffusion is the fastest, and the thermal runaway monomers are the most; at point -2 (the edge of the midpoint), the thermal runaway time $t_{n}$ is the second, and the thermal runaway battery $P_{N}$ is the same as that at point-1, and the thermal runaway monomers are the most; point- When triggered at 3,4 , and 5 , the thermal runaway time $t_{n}$ is longer and not much different, but the thermal runaway battery ratio $P_{N}$ at point- 4 is the same as that at points- 1 and 2 . 
Table 1. Single point hot trigger PN, tn result table for different positions of module

\begin{tabular}{cccc}
\hline Serial number & Thermal trigger battery position & $\begin{array}{c}\text { Thermal runaway time } \\
t_{n} / \mathrm{s}\end{array}$ & $\begin{array}{c}\text { Percentage of thermal } \\
\text { runaway batteries } P_{N}\end{array}$ \\
\hline 1 & Geometric angle position (point-1) & 458 & $25 \%$ \\
2 & Edge midpoint position (point-2) & 818 & $25 \%$ \\
3 & Surface centre position (point-3) & 1232 & $16.7 \%$ \\
4 & Position B 1/4 from opposite side (point-4) & 1283 & $25 \%$ \\
5 & Position C 1/4 from opposite side (point-5) & 1202 & $16.7 \%$ \\
\hline
\end{tabular}

\subsection{Simulation of thermal runaway of module under multi-point trigger}

In order to study the influence of trigger points on the thermal runaway rule of the module, the simulation experiment is a combination test of the above five points. In order to better reflect the law of thermal runaway at module level under multi-point trigger, the multi-point thermal trigger experiment is set up with a smaller heat source, and other conditions are the same as the singlepoint trigger experiment.

(1) Two-point thermal trigger

When the number of thermal trigger points is 2 , there

are a total of $C_{5}^{2}=10$ test methods. Take the combination point $(1,2)$ as an example, and simultaneously apply a point heat source to the point- 1 and point- 2 positions to simulate thermal runaway, Table 2 is the module-level two-point thermal trigger $P_{N}, t_{n}$ results table, the combination point $(1,2)$ the fastest thermal runaway, where $t_{n}=871.78 \mathrm{~s}$, but only one thermal runaway monomer, that is, $N=1, P_{N}=8.33 \%$; the combination point $(2,3),(1,4),(2,5)$ The highest number of thermal runaway monomers, i.e., $N=2$ and $P_{N}=16.67 \%$; the combination points $(2,4)$ and $(3,4)$ both have monomers that reach the temperature condition, but not the temperature rise rate condition, so the number of thermal runaway monomers $N=0$.

Table 2. Module two-point hot trigger PN, tn result table

\begin{tabular}{ccc}
\hline $\begin{array}{c}\text { Thermal trigger } \\
\text { point }\end{array}$ & Thermal runaway time $t_{n} / \mathrm{s}$ & $\begin{array}{c}\text { Percentage of thermal runaway } \\
\text { batteries } P_{N}\end{array}$ \\
\hline$(1,2)$ & 871.78 & $8.33 \%(N=1)$ \\
$(2,3)$ & 1942.72 & $16.67 \%(N=2)$ \\
$(1,3)$ & 1204.8 & $8.33 \%(N=1)$ \\
$(1,4)$ & 1103.2 & $16.67 \%(N=2)$ \\
$(1,5)$ & 1215.6 & $8.33 \%(N=1)$ \\
$(2,4)$ & None (at 934.07s the temperature condition is reached, but the & $0(N=0)$ \\
$(2,5)$ & temperature rise rate condition is not reached) \\
$(3,4)$ & 1188.9 & $16.67 \%(N=2)$ \\
$(3,5)$ & None (at $600.94 \mathrm{~s}$ the temperature condition is reached, but the & $0(N=0)$ \\
$(4,5)$ & temperature rise rate condition is not reached) & $8.33 \%(N=1)$ \\
& 1099.5 & $8.33 \%(N=1)$
\end{tabular}

(2) Three- and four-point heat trigger

The following simulation experiments will be performed on three-point thermal triggering. There are $\mathrm{C}_{5}^{3}=10$ and $\mathrm{C}_{5}^{4}=5$ combinations, which are the same as the two-point thermal triggering experiment. The same size heat source and boundary conditions are set. Perform simulation experiments on 10 sets of combined points. Table 3 shows the results of module-level three-point thermal trigger $P_{N}$ and $t_{n}$. It can be seen that when the combination point $(1,3,5)$ is triggered, the thermal runaway occurs fastest, in which $t_{n}=775.15 \mathrm{~s}$, and the number of thermal runaway monomers is the largest, that is, $N=7, P_{N}=58.33 \%$; at the combination point Thermal triggering at $(1,2,3)$ and $(1,2,4)$, although thermal runaway is not the fastest, the number of thermal runaway monomers can reach the maximum, namely $N=7, P_{N}$ $=58.33 \%$.

Table 3. Module three-point hot trigger PN, tn result table

\begin{tabular}{ccc}
\hline $\begin{array}{c}\text { Thermal trigger } \\
\text { point }\end{array}$ & Thermal runaway time $t_{n} / \mathrm{s}$ & $\begin{array}{c}\text { Percentage of thermal runaway } \\
\text { batteries } P_{N}\end{array}$ \\
\hline$(1,2,3)$ & 1207.46 & $58.33 \%(N=7)$ \\
$(1,2,4)$ & 1099.83 & $58.33 \%(N=7)$ \\
$(1,2,5)$ & 1217.01 & $41.67 \%(N=5)$ \\
$(1,3,4)$ & 1080.32 & $25 \%(N=3)$ \\
$(1,3,5)$ & 775.15 & $58.33 \%(N=7)$ \\
$(1,4,5)$ & 1084.05 & $50 \%(N=6)$
\end{tabular}




\begin{tabular}{ccc}
$(2,4,5)$ & 1107.67 & $33.33 \%(N=4)$ \\
$(2,3,5)$ & 1110.92 & $50 \%(N=6)$ \\
$(2,4,5)$ & 1107.67 & $33.33 \%(N=4)$ \\
$(3,4,5)$ & 1475.72 & $33.33 \%(N=4)$ \\
\hline
\end{tabular}

Table 4 is the result table of module-level four-point thermal triggering $P_{N}$ and $t_{n}$. Thermal triggering at the combination point $(1,2,3,4)$, the battery module can experience thermal runaway fastest, but the proportion of thermal runaway batteries $P_{N}=66.67 \%(N=8)$ is not the highest; and when the combination point $(1,2,3,5)$ is triggered, the proportion of thermal runaway batteries $P_{N}$ $=75 \%(N=9)$ reaches the maximum.

Table 4. Module four-point hot trigger PN, tn result table

\begin{tabular}{ccc}
\hline $\begin{array}{c}\text { Thermal trigger } \\
\text { point }\end{array}$ & Thermal runaway time $t_{n} / \mathrm{s}$ & $\begin{array}{c}\text { Percentage of thermal runaway } \\
\text { batteries } P_{N}\end{array}$ \\
\hline$(1,2,3,4)$ & 1055.71 & $66.67 \%(N=8)$ \\
$(1,2,3,5)$ & 1067.73 & $75 \%(N=9)$ \\
$(1,2,4,5)$ & 1071.14 & $58.33 \%(N=7)$ \\
$(1,3,4,5)$ & 1062.68 & $66.67 \%(N=8)$ \\
$(2,3,4,5)$ & 2128.46 & $25 \%(N=3)$ \\
\hline
\end{tabular}

It can be seen that in the single-point thermal trigger experiment, thermal runaway occurs fastest at the geometric angle position (point-1), followed by the edge midpoint (point-2), and the proportion of thermal runaway batteries $P_{N}$ is the largest; Under the thermal trigger of the same number of points, the combination points $(1,2)$, $(1,3,5),(1,2,3,4)$ respectively appear the fastest thermal runaway, and the combination point $(1,3,5)$ The proportion of thermal runaway batteries $P_{N}$ is the largest; the number of thermal trigger points and the proportion of thermal runaway batteries $P_{N}$ show a positive correlation.

\section{Conclusions}

The thesis takes 18650 lithium battery modules as the object, considers the external adiabatic conditions of the battery, combines the thermal abuse model of the lithium battery, and builds the 18650 lithium battery module based on the COMSOL finite element analysis software. According to the American standard SAE J2464-2009 recommended thermal trigger point, the paper carried out single-point and multi-point thermal trigger simulation experiments.

(1) Through the single-point thermal trigger simulation experiment on the five points recommended by SAE $\mathrm{J} 2464-2009$, it is pointed out that the thermal runaway time $t_{n}$ of the geometrical angular position (point-1) is the smallest, the thermal runaway battery $P_{N}$ is the largest, and multi-point combination is performed Thermal trigger simulation experiment, it is clear that the number of thermal trigger points is positively correlated with $P_{N}$;

(2) The electric vehicle battery system also contains many other components. How to build a battery systemlevel model and explore the laws of thermal trigger points, heat dissipation conditions and other factors on the local thermal runaway of the battery system are current shortcomings and issues that still need to be studied in the future.

\section{Acknowledgments}

This paper is one of the stage results of the Guangdong
Key Areas R\&D Project Power Battery System Testing and Evaluation Technology Research (2019B090908003).

\section{References}

1. Feng X, He X, Ouyang M.(2018) A Coupled Electrochemical-Thermal Failure Model for Predicting the Thermal Runaway Behavior of Lithium-Ion Batteries. Journal of The Electrochemical Society, 165(16): A3748-A3765.

2. Sara A, Martin P, Amandine L, et al. (2018) Combined experimental and modeling approaches of the thermal runaway of fresh and aged lithiumion batteries. Journal of Power Sources, 399:264273.

3. Ji C, Sun j, Wang S.F, Wang B, Pan S.(2019) Experimental Research on the Thermal Safety of 32650 Single Cell and Module. Journal of Beijing University of Technology, 45(08):780-786.

4. Christophe Forgez, Dinh Vinh Do, Guy (2009) Thermal modeling of a cylindrical LiFePO 4 /graphite lithium-ion battery. Journal of Power Sources, 2009,195(9).

5. Capron O, Samba A, Omar N, et al (2014) Battery thermal modelling - Assessment of heat distribution and optimization of battery design concept. International Conference on Renewable Energy Research \& Applications. IEEE, 2014.

6. Chen H, Xiong H, Li Y.J. (2019) Research progress on heat generation characteristics of lithium-ion batteries. Energy Storage Science and Technology, 2019, 8(S1): 49-55.

7. Feng X.N, OuYang M.G, Xiang L, Lan G.L, Xia Y, He X.M.(2018) Thermal runaway mechanism of lithium ion battery for electric vehicles: A review. Energy Storage Materials, 10:246-267.23

8. Liu Q.Q. (2018) Research on battery thermal runaway and its expansion characteristics under heating conditions. Hefei University of Technology. 
9. Bernardi D.(1985) A general energy balance for battery systems. Electrochem Soc, 132:5.

10. Lin H, Zhang H.X, Zhao Q.H. (2019) Threedimensional electrochemical-thermal coupling model and heat generation analysis of lithium ion battery. Power Technology, 43(10):1630-1632+1698.

11. Feng Y, Zheng L.L, Dai Z.Q.(2019) The progress of lithium-ion battery simulation models. Energy storage science and technology, 2019,8(S1):18-22.

12. Gu WB, Wang CY.(2000) Thermal-electrochemical modeling of battery systems. Electrochem Soc, 147:2910. 